\title{
Prognostic Significance of Preoperative Serum Uric Acid-to-Lymphocyte Ratio in Hepatocellular Carcinoma Patients Undergoing Postoperative Transarterial Chemoembolization: A Propensity Score-Matching Study
}

\section{Shan-shan Hu}

The First Affiliated Hospital of Chongqing Medical University

\section{Li-heng Liu}

The First Affiliated Hospital of Chongqing Medical University

\section{Li-ping Tang ( $547053591 @ q q . c o m$ )}

The First Affiliated Hospital of Chongqing Medical University https://orcid.org/0000-0003-2524-7483

\section{Research}

Keywords: Hepatocellular carcinoma, Uric acid, Lymphocyte, Inflammation, Immune response, Prognosis

Posted Date: October 9th, 2020

DOl: https://doi.org/10.21203/rs.3.rs-88882/v1

License: (1) (1) This work is licensed under a Creative Commons Attribution 4.0 International License. Read Full License 


\section{Abstract}

\section{Background}

Hepatocellular carcinoma (HCC) accounts for $85 \%-90 \%$ of primary liver cancers and is the seventh most common cancer worldwide. The purpose of this study is to determine an innovative and effective clinical index for prognosis prediction in HCC patients.

\section{Methods}

A total of 132 patients with HCC were included in this research. A cohort of 72 cases was obtained through propensity score-matching. Patients were divided into high- and low-level groups based on the ratio of preoperative uric acid levels to lymphocytes. The differences in clinical characteristics and survival indicators were compared between the two groups.

\section{Results}

There was a statistical difference between uric acid-to-lymphocyte ratio (ULR) level and tumor size $(P<$ $0.01)$. Multivariate analysis showed that ULR $(P=0.03)$ and tumor size $(P=0.03)$ were independent risk factors for overall survival (OS). ULR $(P<0.04)$ and diabetes $(P=0.04)$ were independent risk factors for progression-free survival (PFS) in HCC patients. Further survival analysis of the entire cohort and propensity score-matching cohort showed that the OS and PFS in the high-level ULR group were significantly shorter than those in the low-level ULR group (log-rank $P<0.001)$.

\section{Conclusion}

This study demonstrated that ULR is a marker of poor prognosis in postoperative HCC patients, while high ULR levels predicted shorter OS and PFS.

\section{Background}

Primary liver cancer is one of the most common cancer types, which ranks as the third leading cause of cancer-related death globally[1]. Physiologically, primary liver cancer can be classified into three subtypes, including hepatocellular carcinoma (HCC), intrahepatic cholangiocarcinoma, and combined hepatocellular-cholangiocarcinoma. HCC accounts for $75-85 \%$ of all subtypes. Previous reports have estimated that the incidence of $\mathrm{HCC}$ by 2030 will continue to rise in some countries, such as China and the United States, while gradually declining in other countries like Japan[2]. A recent prospective study has confirmed that transarterial chemoembolization (TACE) can significantly prolong the overall survival (OS) and progression-free survival (PFS) in postoperative HCC patients at medium and high risk of recurrence[3]. Although adjuvant therapy for HCC, such as TACE, radio-frequency ablation, radiotherapy, and chemotherapy, has been greatly developed in recent years, the postoperative recurrence and mortality rate remain high. Effective and convenient monitoring and management of postoperative patients is still a significant problem that needs to be solved urgently. A dynamic change in alpha-fetoprotein (AFP) can 
be observed clinically, but the diagnostic and prognostic values are limited due to their lack of specificity and stability. Therefore, it is particularly important to identify a stable and simple serological index to effectively predict the prognosis in postoperative HCC patients.

Serum uric acid (SUA) is synthesized by xanthine dehydrogenase, which is the final product of purine nucleoside metabolism and exists in cells, tissues, and organs. It is generally believed that uric acid is an indicator of tumor cell death. When cells die, a large amount of DNA and RNA in the whole nucleus and cytoplasm is degraded, most of which will be converted into SUA. SUA then reacts with reactive oxygen species, nitricoxide, and reactive nitrogen species and participates in oxidation and pro-inflammatory activity, which is related to cell transformation and proliferation[4]. In addition, hyperuricemia may lead to hypertriglyceridemia, insulin resistance, hyperinsulinemia, and hyperglycemia, which promote pathogenesis of metabolic syndrome and is conducive to tumor occurrence and chronic inflammation. Clinically, cancer growth is often accompanied by cell death and metabolic syndrome. Related studies have also confirmed that elevated SUA levels can increase the risk of morbidity and mortality in many cancers, such as gastric cancer[7], colorectal cancer[8], renal cell carcinoma[9], and liver cancer[10]. Elevated SUA level is widely regarded as a risk factor for poor cancer prognosis.

Lymphocytes are produced by lymphoid tissue and act as an important part of the immune response. Lymphocytes are separated into T, B, and NK cells according to their origin, surface molecules, and functions. Inflammation and immune response in the tumor microenvironment are related to tumor growth and metastasis. Many studies have confirmed that lymphocytes perform cancer monitoring via their immune function. They are also related to prognosis of many cancers, such as liver[12], colorectal[13], pancreatic[14, 15], and breast[16]. Moreover, systemic inflammatory response is often accompanied by an increase in neutrophil and monocyte count and a sharp decrease in lymphocytes. Neutrophils can not only secrete pro-inflammatory factors such as growth factor- $\beta$, leading to significant immunosuppressive effect, but also inhibit lymphocyte cytolytic activity to promote angiogenesis and tumor growth[16]. These studies have all shown that lymphopenia is closely related to increased inflammation and immunodeficiency in tumors, which may be a risk factor for tumor growth and invasion.

Followed by the recent emergence of a large number of biological indicators, such as platelet-tolymphocyte ratio, neutrophil-to-lymphocyte ratio, and uric acid-to-lymphocyte ratio (ULR), tumor prognosis has become the focus of many research studies. Since multiple factors, including metabolism, inflammation, and immune regulation, are often related to tumor occurrence and progression, ULR may act as an effective tumor prognostic marker. The present study is the first to explore the prognostic significance of ULR in postoperative HCC patients.

\section{Methods}

\subsection{Study design and project}


This was a single-center, retrospective study involving 132 patients with HCC diagnosed by postoperative pathological examination in the first affiliated Hospital of Chongqing Medical University (Chongqing, China) between 2012 and 2017. The study was approved by the Ethics Committee of Chongqing Medical University (ID: 2020-431).

\subsection{Inclusion and exclusion criteria}

Appropriate patients were included in accordance with the following eligibility criteria:

i. Patients who were pathologically diagnosed with $\mathrm{HCC}$ after surgery and whose clinical stage was Barcelona Clinic Liver Cancer (BCLC) A-B.

ii. Once postoperative TACE ( $p$-TACE) was performed.

iii. Clinical and follow-up data were complete.

iv. No other tumors were present.

v. Radiotherapy, chemotherapy, radiofrequency ablation, and targeted immunotherapy were not performed before and after operation.

vi. Patients with gout, hematological diseases, kidney diseases, drug poisoning, and other diseases affecting SUA and blood lymphocyte count were excluded.

\subsection{Data collection and method definition}

\section{Date collection:}

Data were collected from 132 eligible patients, including gender, age, body mass index (BMI), tumor size, tumor stage, operation method, peripheral serum index, and accompanying diseases (such as cardiocerebrovascular disease and diabetes). Moreover, survival data were obtained from regular follow-up after surgery.

\section{Laboratory markers with grouping criteria and outcome date}

Peripheral serum biological indicators included preoperative SUA, blood lymphocyte count, serum albumin, and AFP. According to the hospital's clinical standard, SUA at 208-428 mmol/L for males and 155-357 mmol/L for females (postmenopausal females had the same criteria as men), lymphocyte count at 1.10-3.2 $\times 10^{9} / \mathrm{L}$, albumin $>4 \mathrm{~g} / \mathrm{dL}$, and AFP $\leq 400 \mathrm{ng} / \mathrm{mL}$ were defined as the normal ranges. ULR was defined as the ratio of SUA $(\mathrm{mg} / \mathrm{dL})$ to lymphocytes $\left(\times 10^{9} / \mathrm{L}\right)$. Concomitant cardiovascular and cerebrovascular diseases were defined as at least one type of disease, such as hypertension, coronary artery disease, heart failure, aortic aneurysm, and stroke. Diabetes categories included type 1, type 2 , and special types of diabetes. Finally, patients were followed up for up to three years by telephone every six months until death or loss of contact. OS was calculated from operation to death or until follow-up deadline. PFS was calculated from operation to relapse or death or until follow-up deadline.

\section{Method definition}




\section{Intervention}

To reduce the impact of different treatment options on patient prognosis, early and middle stage HCC patients who underwent partial resection combined with at least one p-TACE were chosen. The p-TACE was performed to reduce early HCC relapse rate. Generally, 5-F catheter or microcatheter was inserted into the proper hepatic artery via the right femoral artery guided by digital subtraction angiography.

Chemotherapy drugs were then slowly injected, followed by lipiodol $(2-5 \mathrm{~mL})$ embolization. It should be noted that each patient's treatment plan was slightly different and was usually completed within two weeks to two months after surgery.

\section{Cut-off ULR value}

Time-dependent receiver operating characteristic (ROC) curve was used to determine the cut-off ULR value. Patients were divided into high-level (ULR > 5.47) and low-level (ULR $\leq 5.47)$ groups. The two groups' clinical features and survival outcomes were then compared.

\section{Propensity score-matching model}

Propensity score-matching (PSM) model was used to reduce the influence of confounding factors. Age, gender, BMI, hepatitis B virus (HBV) infection, tumor size, AFP, liver cirrhosis, cardio-cerebrovascular disease, and diabetes were included in the propensity score. The 1:1 paired model was established using the caliper nearest the matching algorithm and specifying the distance at 0.20 SD of the logit of PS. Finally, 72 cases (36 pairs) were enrolled in the PSM cohort.

\subsection{Statistical method}

SPSS 26.0 and R 3.6 .3 software (https://www.r-project.org/) were utilized to perform statistical analysis. Time-dependent ROC curve was used to determine the ULR cut-off value. Pearson's $X^{2}$ or Fisher's exact test was used to analyze the correlation between ULR level and clinicopathological characteristics. Cox regression was used to calculate independent risk factors for postoperative prognosis. Kaplan-Meier and log-rank tests were applied to evaluate survival of the entire and PSM cohorts.

\section{Results}

\subsection{Baseline}

A total of 203 patients underwent partial resection and p-TACE. Among them, two cases received a liver transplant, four cases received intravenous systemic chemotherapy after operation, 25 cases had gout, hematological diseases, kidney diseases, and drug poisoning, and 40 cases were lost to follow-up. A total of 132 cases were included in the study (Figure 1).

There were 111 males (84.1\%) and 21 females (15.9\%) in the entire cohort. The average age was 53.0 (range: 13-83) years and average BMI was 22.55 (range: $16.5-32.0) \mathrm{kg} / \mathrm{m}^{2}$. The average tumor size was 
5.87 (range: $0.5-18$ ) cm, average AFP was 13816.2 (range: $0.24-484000$ ) $\mathrm{ng} / \mathrm{mL}$, average SUA was 317.64 (range: $92-684$ ) $\mathrm{mmol} / \mathrm{L}$, average lymphocyte count was 1.40 (range: $0.33-9.10$ ) $\times 10^{9} / \mathrm{L}$, and average albumin was 41 (range: $21-55) \mathrm{g} / \mathrm{L}$. There were 114 (86.4\%) cases with HBV infection, 82 (62.1\%) with liver cirrhosis, 18 (13.6\%) with cardio-cerebrovascular disease, and 11 (8.3\%) with diabetes (Table 1).

There were 61 males (84.7\%) and 11 females (15.3\%) in the PSM cohort. The average age was 53.8 (range: 19-83) years, average BMI was 22.43 (range: $16.49-32.0$ ) kg/m², average tumor size was 6.90 (range: $0.5-18$ ) cm, average AFP was 19385.96 (range: $1.21-484000) \mathrm{ng} / \mathrm{mL}$, average SUA was 329.17 (range: $126-684$ ) $\mathrm{mmol} / \mathrm{L}$, average lymphocyte count was 1.23 (range: $0.33-2.41$ ) $\times 10^{9} / \mathrm{L}$, and average albumin was 40.64 (range: $21-55) \mathrm{g} / \mathrm{L}$. There were 62 (86.1\%) cases with HBV infection, 45 (62.5\%) with liver cirrhosis, five (6.9\%) with cardio-cerebrovascular disease, and three (4.2\%) with diabetes (Table 1$)$.

Postoperative survival data showed that the average follow-up time was 24 (range: $2-36$ ) months. The three-year recurrence and mortality rates were both 64 (47.8\%).

\subsection{Determination of optimal ULR cut-off value}

The cut-off value (ULR $=5.47$ ) was selected at the most approximate index on the ROC curve (Figure 2). All subjects were divided into high-level (ULR > 5.47) and low-level $(U L R \leq 5.47)$ groups based on this cutoff value. The average ULR value was 4.65 (range: $0.6-13.84$ ) in the entire cohort. A total of $40(30.3 \%)$ cases were included in the high-level group with an average ULR value of 7.60 (range: $5.49-13.84$ ). A total of $92(69.7 \%)$ cases were included in the low-level group with an average ULR value of 3.37 (range: 0.6-5.47). The average ULR value in the PSM model was 5.41 (range: 1.5-13.84). The average ULR values in the high- and low-level groups were 7.69 (5.49-13.84) and $3.13(1.50-5.31)$, respectively.

\subsection{ULR level and clinical features}

Statistical analysis was performed between ULR level and HCC patient clinical characteristics. The proportion of tumors sized $>5 \mathrm{~cm}$ accounted for $65 \%$ of participants in the high-level group and $32.6 \%$ in the low-level group. A statistically significant difference was present between the ULR level and tumor size $(P<0.01)$. However, there was no statistical difference between the ULR level and age, gender, $B M I$, HBV infection, AFP, liver cirrhosis, cardiovascular disease, or diabetes $(P>0.05$, Table 1$)$.

\subsection{Univariate and multivariate analysis}

Cox regression analysis was used to predict independent risk factors for postoperative survival. All elements with $\mathrm{P}<0.2$ in the univariate analysis were used in the multivariate analysis, where $\mathrm{P}<0.05$ was considered statistically significant.

Univariate analysis indicated a significant difference between tumor size $(P<0.01)$, lymphocyte count $(P$ $<0.05)$, ULR level $(P<0.001)$, and OS. There were also significant differences between tumor size $(P<$ 0.01) and ULR $(P<0.001)$ and PFS. Other clinical features, such as age, gender, BMI, HBV infection, AFP, 
uric acid, liver cirrhosis, cardiovascular disease, and diabetes, were not significantly correlated with postoperative survival (Table 2).

Tumor size $(P<0.01)$, lymphocyte count $(P<0.05)$, and ULR level $(P<0.001)$ were further included in the multivariate analysis of OS. The results revealed that tumor size and ULR level can be used as independent risk factors for OS in postoperative patients. Larger tumors $(>5 \mathrm{~cm})$ and higher ULR $(>5.47)$ indicated shorter OS (Table 2).

Gender $(P=0.08)$, tumor size $(P<0.01)$, SUA $(P=0.10)$, lymphocyte count $(P=0.13)$, albumin $(P=0.19)$, ULR $(P<0.001)$, and diabetes $(P=0.08)$ were included in multivariate analysis of PFS. The final results demonstrated that ULR $(P<0.001)$ and diabetes $(P=0.04)$ may be independent risk factors for PFS. Postoperative HCC patients with higher ULR (>5.47) and diabetes relapsed more easily and had a shorter PFS (Table 2).

\subsection{Survival analysis}

In the entire cohort, the average postoperative follow-up time was 20.67 (range: 2-36) months, three-year recurrence rate was $27 / 40$ (67.5\%), and three-year mortality rate was $28 / 40(70.0 \%)$ in the high-level group. The average follow-up time was 25.43 (range: 5-36) months, three-year recurrence rate was 37/92 (40.2\%), and three-year mortality rate was 36/92 (39.1\%) in the low-level group. Survival analysis showed that high-level group OS and PFS were significantly shorter than those in the low-level group (log-rank $\mathrm{P}<$ 0.001 ; Figure 3A-B).

In the PSM cohort, the average postoperative follow-up time was 20.67 (range: 2-36) months, three-year recurrence rate was $24 / 36$ (66.7\%), and three-year mortality rate was $25 / 36(69.4 \%)$ in the high-level group. The average follow-up time was 25 (range: 5-36) months, three-year recurrence rate was 14/36 (38.9\%), and three-year mortality rate was $12 / 36$ (33.3\%) in the low-level group. Survival analysis showed that high-level group OS and PFS were significantly shorter than those in the low-level group (log-rank $P<0.001$; Figure 3C-D).

\section{Discussion}

$\mathrm{HCC}$ is the second leading cause of death in males and sixth in females and a common threat to human health around the world. Previous studies have found that uric acid has the dual anti- and pro-oxidant effects. Therefore, the relationship between SUA and cancer is controversial. However, there is a growing consensus that high SUA levels increase the risk of cancer. When intracellular SUA increases, inflammatory stress is induced by affecting the activation of cyclooxygenase- 2 and production of reactive oxygen species, while extracellular SUA level increase stimulates a variety of transcription factors and promotes cancer cell survival, proliferation, and migration[7, 9, 21]. SUA also enters adipocytes through uric acid-specific transporter and down-regulates expression of xanthine dehydrogenase or xanthine oxidoreductase, thus increasing expression of matrix metalloproteinases, cyclooxygenase-2, epithelial-mesenchymal transition genes, and transforming growth factor- $\beta$ to encourage stronger 
invasiveness and metastatic ability in liver, breast, and other cancer cells[5, 17, 18]. Recent prospective cohort studies have also shown that some uric acid-lowering drugs, such as allopurinol[19] and colchicine[20], reduce the risk of liver and prostate cancers. All of these findings demonstrate that even if the physiological SUA level can prevent aging and cancer caused by oxidants and free radicals, once SUA level increases, its pro-oxidation and pro-inflammatory effects have a more far-reaching impact on the tumor.

In addition, lymphocytes also play a key role in tumor immune surveillance and tumor cell defense. Lymphocytes, such as B, T (helper T cell and cytotoxic T cell,), and NK cells, eliminate tumor cells by producing antibodies, recognizing tumor antigens, and secreting cytokines, thus inhibiting tumor proliferation and invasion[12, 15,22]. Many studies have reported that an increase in lymphocyte level is related to better prognosis in some tumors, such as liver[12, 24], pancreatic[14, 15], and gastric[22, 23] cancers. The main mechanism may affect tumor micro-environment immune and inflammatory response. On one hand, lymphocytes improve host anti-cancer immunity, induce cytotoxic cell death, and inhibit tumor cell proliferation and migration[22]. On the other hand, a decrease in lymphocytes is often accompanied by systemic inflammation, which produces oxygen free radicals and various inflammatory cytokines, thus stimulating tumor proliferation and metastasis[24]. At the same time, tumor cells also produce tumor factors and pro-inflammatory mediators, increase peripheral blood neutrophils and monocytes, and reduce lymphocyte count. Thus, peripheral blood cell components are often used to predict tumor occurrence and progression. Based on the changes in inflammation, immune response, and metabolism in tumor cell micro-environment, ULR has been proposed as a new prognostic indicator. However, it has only been verified in early lung cancer[25]. Compared to a single index of inflammation, metabolism, or immunity, ULR is more comprehensive, representative, and consistent with tumor characteristics.

To the best of our knowledge, this is the first study to propose that ULR may be related to HCC tumor size and that it may be an independent risk factor for prognosis in early-middle stage HCC patients after combined surgery and p-TACE. Survival analysis also showed that ULR $>5.47$ predicted shorter OS and PFS. The PSM model was used to confirm the above results.

\section{Limitations}

This study was a retrospective study, so the timing of TACE treatment, specific chemotherapy drugs, and times were inconsistent, while complications and causes of death during follow-up were not recorded in detail. This may lead to a deviation in the prognosis outcome. Second, contact was lost with some patients due to long follow-up time, which reduced the sample size and increased the statistical error. Finally, it should be noted that all study results were obtained from a small sample size. Thus, prospective studies with a larger sample size and strict variable control are still needed to further confirm these results. Moreover, the specific mechanism requires more in vivo and in vitro experiments to explore.

\section{Conclusions}


The present study demonstrated that peripheral ULR is an independent risk factor for prognosis in patients with early and middle stage HCC undergoing partial resection and p-TACE. High ULR level was shown to be a sign of a larger tumor size and poor prognosis, indicating shorter OS and PFS.

\section{List Of Abbreviations}

\begin{tabular}{|llll|}
\hline hepatocellular carcinoma & HCC & body mass index & BMI \\
\hline uric acid-to-lymphocyte ratio & ULR & receiver operating characteristic & ROC \\
\hline overall survival & OS & propensity score-matching & PSM \\
\hline progression-free survival & PFS & hepatitis B virus & HBV \\
\hline transarterial chemoembolization & TACE & postoperative TACE & p-TACE \\
\hline alpha-fetoprotein & AFP & serum uric acid & SUA \\
\hline
\end{tabular}

\section{Declarations}

\section{Ethics approval and consent to participate}

This retrospective study was approved by the Ethics Committee of Chongqing Medical University (ID: 2020-431; Chongqing, China).

\section{Consent for publication}

All authors have agreed with submission to WORLD J SURG ONCOL.

\section{Availability of data and materials}

The datasets used and/or analyzed are available from the corresponding author on reasonable request.

\section{Competing interests}

The authors declare that they have no competing interests.

\section{Funding}

Not applicable.

\section{Authors' contributions}

SH and LL carried out the studies and participated in collecting data, statistical analysis, and drafting the manuscript. LT participated in data acquisition, analysis, and interpretation. All authors read and approved the final manuscript. 
Acknowledgements

Not applicable.

\section{References}

1. Bray F, Ferlay J, Soerjomataram I, Siegel RL, Torre LA, Jemal A. Global cancer statistics 2018 : GLOBOCAN estimates of incidence and mortality worldwide for 36 cancers in 185 countries. CA Cancer J Clin. 2018; 68:394-424.

2. Liu WR, Tian MX, Tao CY, Tang Z, Zhou YF, Song SS, et al. Adjuvant Transarterial chemoembolization does not influence recurrence-free or overall survival in patients with combined hepatocellular carcinoma and Cholangiocarcinoma after curative resection: a propensity score matching analysis. BMC Cancer. 2020; 20:642.

3. Vogel A, Cervantes A, Chau I, Daniele B, Llovet JM, Meyer T, et al. Hepatocellular carcinoma: ESMO Clinical Practice Guidelines for diagnosis, treatment and follow-up.Ann. Oncol. 2018; 29:iv238-iv255.

4. Liu, D, Yun, Y, Yang, D, Hu, X, Dong, X, Zhang, N, et al. What is the biological function of uric acid? an antioxidant for neural protection or a biomarker for cell death. Disease markers. 2019; 2019:4081962.

5. Battelli MG, Bortolotti M, Polito L, Bolognesi A. Metabolic syndrome and cancer risk: The role of xanthine oxidoreductase. Redox Biol. 2019; 21:101070.

6. Dovell F, Boffetta P. Serum uric acid and cancer mortality and incidence: a systematic review and meta-analysis. Eur J Cancer Prev. 2018; 27:399-405.

7. Yang S, He X, Liu Y, Ding X, Jiang H, Tan Y, et al. Prognostic Significance of Serum Uric Acid and Gamma-Glutamyltransferase in Patients with Advanced Gastric Cancer. Dis Markers. 2019; 2019:1415421.

8. Mao L, Guo C, Zheng S. Elevated urinary 8-oxo-7,8-dihydro-2'-deoxyguanosine and serum uric acid are associated with progression and are prognostic factors of colorectal cancer. Onco Targets Ther. 2018; 11:5895-5902.

9. Yim K, Bindayi A, McKay R, Mehrazin R, Raheem OA, Field C, et al. Rising Serum Uric Acid Level Is Negatively Associated with Survival in Renal Cell Carcinoma. Cancers (Basel). 2019; 11:536.

10. Hayashi M, Yamada S, Tanabe H, Takami H, Inokawa Y, Sonohara F, et al. High Serum Uric Acid Levels Could Be a Risk Factor of Hepatocellular Carcinoma Recurrences. Nutr Cancer. 2020; 15:1-8.

11. Yan S, Zhang P, Xu W, Liu Y, Wang B, Jiang T, et al. Serum Uric Acid Increases Risk of Cancer Incidence and Mortality: A Systematic Review and Meta-Analysis. Mediators Inflamm. 2015; 2015:764250.

12. Liu X, He L, Han J, Wang L, Li M, Jiang Y, et al. Association of neutrophil-lymphocyte ratio and T lymphocytes with the pathogenesis and progression of HBV-associated primary liver cancer. PLoS One. 2017; 12:e0170605. 
13. Yoshida D, Minami K, Sugiyama M, Ota M, Ikebe M, Morita M, et al. Prognostic Impact of the Neutrophil-to-Lymphocyte Ratio in Stage I-II Rectal Cancer Patients. J Surg Res. 2020; 245:281-287.

14. Rho SY, Hwang HK, Chong JU, Yoon DS, Lee WJ, Kang CM. Association of preoperative total lymphocyte count with prognosis in resected left-sided pancreatic cancer. ANZ J Surg. 2019; 89:503508.

15. Kawai M, Hirono S, Okada KI, Miyazawa M, Shimizu A, Kitahata Y, et al. Low lymphocyte monocyte ratio after neoadjuvant therapy predicts poor survival after pancreatectomy in patients with borderline resectable pancreatic cancer. Surgery. 2019; 165:1151-1160.

16. Losada B, Guerra JA, Malón D, Jara C, Rodriguez L, Del Barco S. Pretreatment neutrophil/lymphocyte, platelet/lymphocyte, lymphocyte/monocyte, and neutrophil/monocyte ratios and outcome in elderly breast cancer patients. Clin Transl Oncol. 2019; 21:855-863.

17. Fini MA, Elias A, Johnson RJ, Wright RM. Contribution of uric acid to cancer risk, recurrence, and mortality. Clin Transl Med. 2012; 1:16.

18. Chen GL, Ye T, Chen HL, Zhao ZY, Tang WQ, Wang LS, et al. Xanthine dehydrogenase downregulation promotes TGF $\beta$ signaling and cancer stem cell-related gene expression in hepatocellular carcinoma. Oncogenesis. 2017; 6:e382.

19. Shih HJ, Kao MC, Tsai PS, Fan YC, Huang CJ. Long-term allopurinol use decreases the risk of prostate cancer in patients with gout: a population-based study. Prostate Cancer Prostatic Dis. 2017; 20:328-333.

20. Kuo MC, Chang SJ, Hsieh MC. Colchicine Significantly Reduces Incident Cancer in Gout Male Patients: A 12-Year Cohort Study. Medicine (Baltimore). 2015; 94:e1570.

21. Yue CF, Feng PN, Yao ZR, Yu XG, Lin WB, Qian YM, et al. High serum uric acid concentration predicts poor survival in patients with breast cancer. Clin Chim Acta. 2017; 473:160-165.

22. Chen L, Hao Y, Cong X, Zou M, Li S, Zhu L, et al. Peripheral Venous Blood Platelet-to-Lymphocyte Ratio (PLR) for Predicting the Survival of Patients With Gastric Cancer Treated With SOX or XELOX Regimen Neoadjuvant Chemotherapy. Technol Cancer Res Treat. 2019; 18:1533033819829485.

23. Shen Q, Liu W, Quan H, Pan S, Li S, Zhou T, et al. Prealbumin and lymphocyte-based prognostic score, a new tool for predicting long-term survival after curative resection of stage II/III gastric cancer. $\mathrm{Br} \mathrm{J}$ Nutr. 2018; 120:1359-1369.

24. Ha SY, Choi S, Park S, Kim JM, Choi GS, Joh JW, et al. Prognostic effect of preoperative neutrophillymphocyte ratio is related with tumor necrosis and tumor-infiltrating lymphocytes in hepatocellular carcinoma. Virchows Arch. 2020. doi: 10.1007/s00428-020-02841-5.

25. Yang Z, Li S, Zhao L, Lv W, Ju J, Zhang W, et al. Serum uric acid to lymphocyte ratio: A novel prognostic biomarker for surgically resected early-stage lung cancer. A propensity score matching analysis. Clin Chim Acta. 2020; 503:35-44.

\section{Tables}


Due to technical limitations, table PDFs are only available as a download in the Supplemental Files section.

\section{Figures}

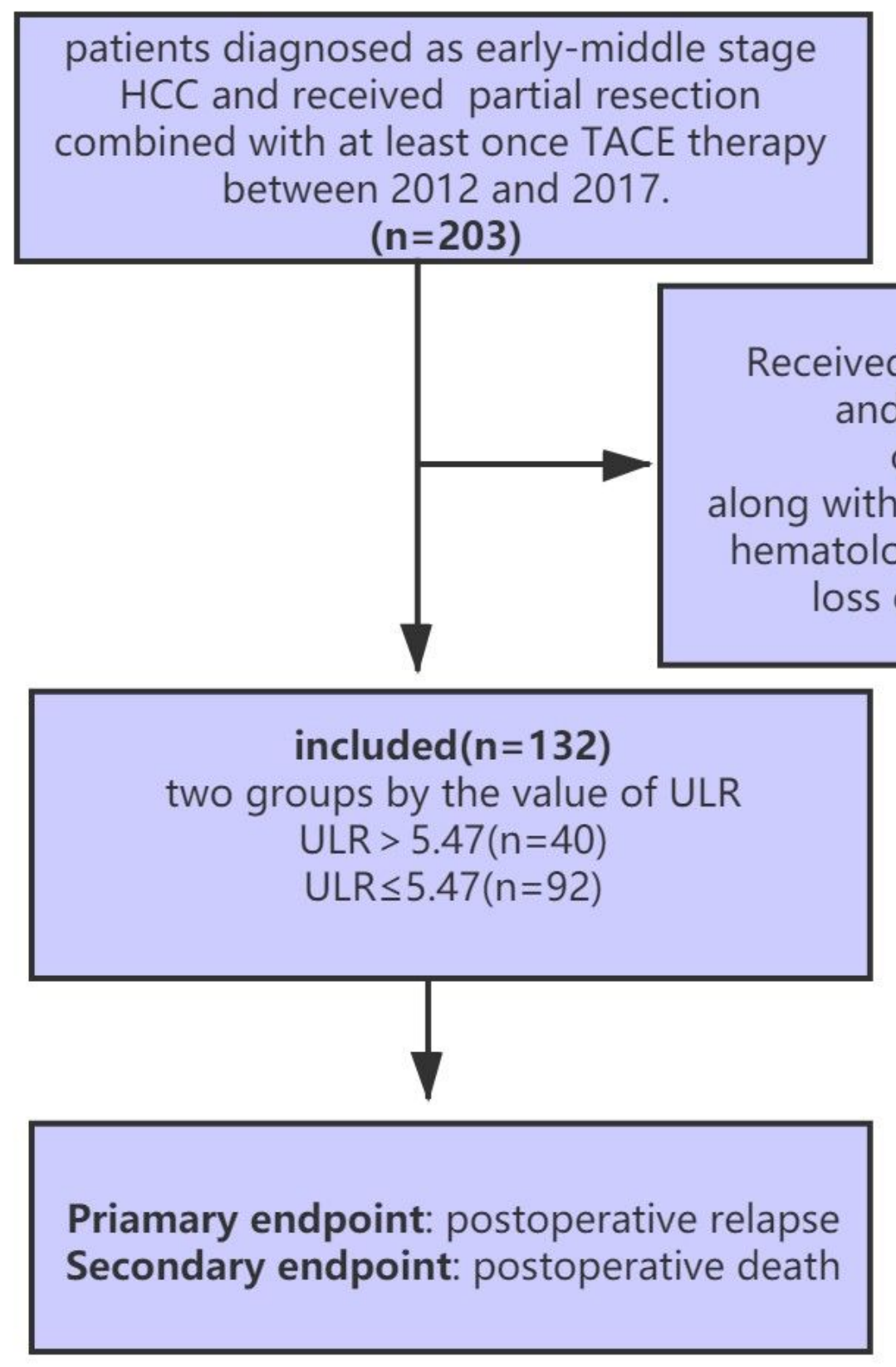

\section{Figure 1}

Case collection flowchart. 


\section{3-year survival ROC}

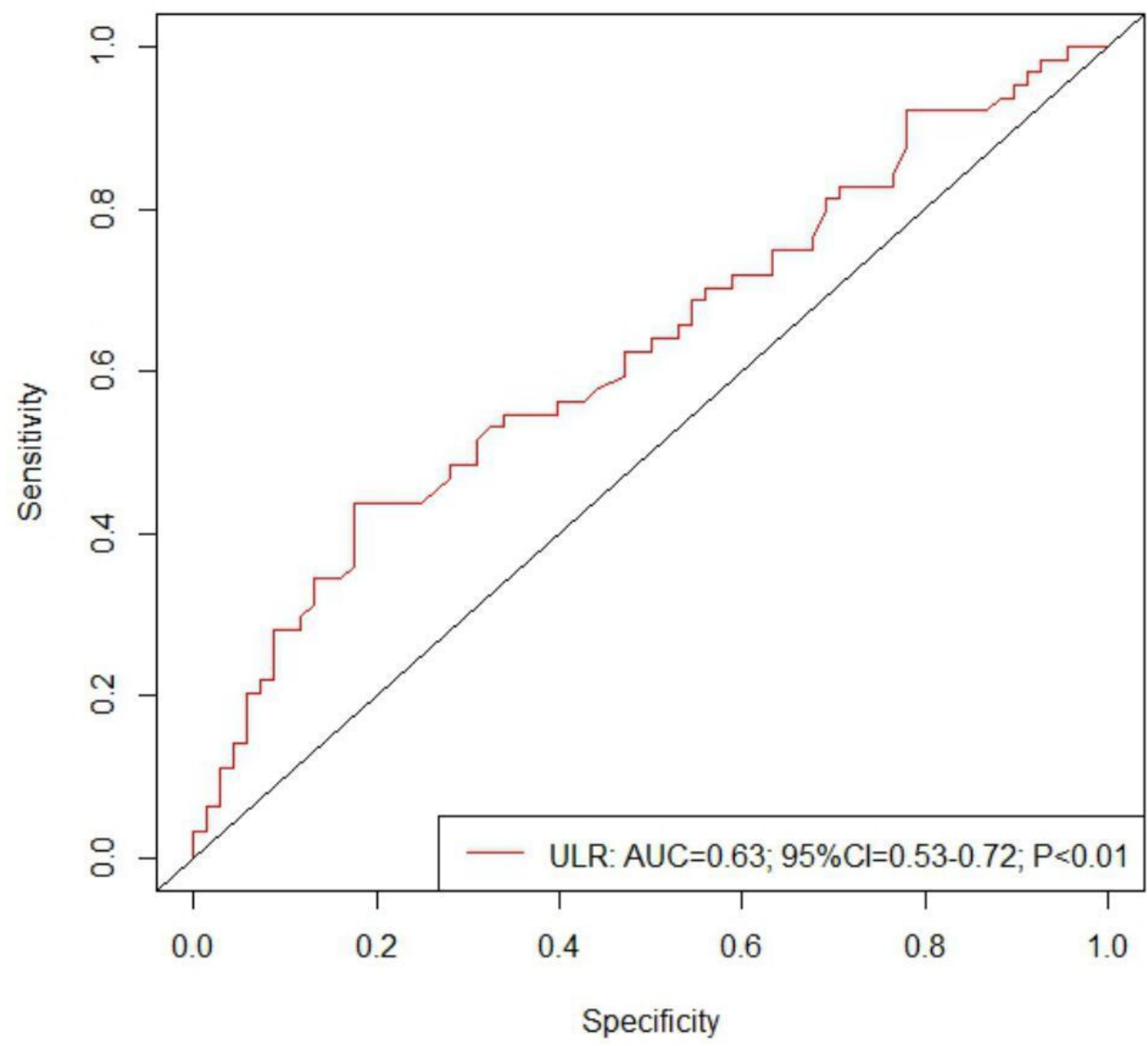

Figure 2

Time-dependent ROC curves for survival analysis. Note: the optimal cut-off value was selected at an approximate index. 

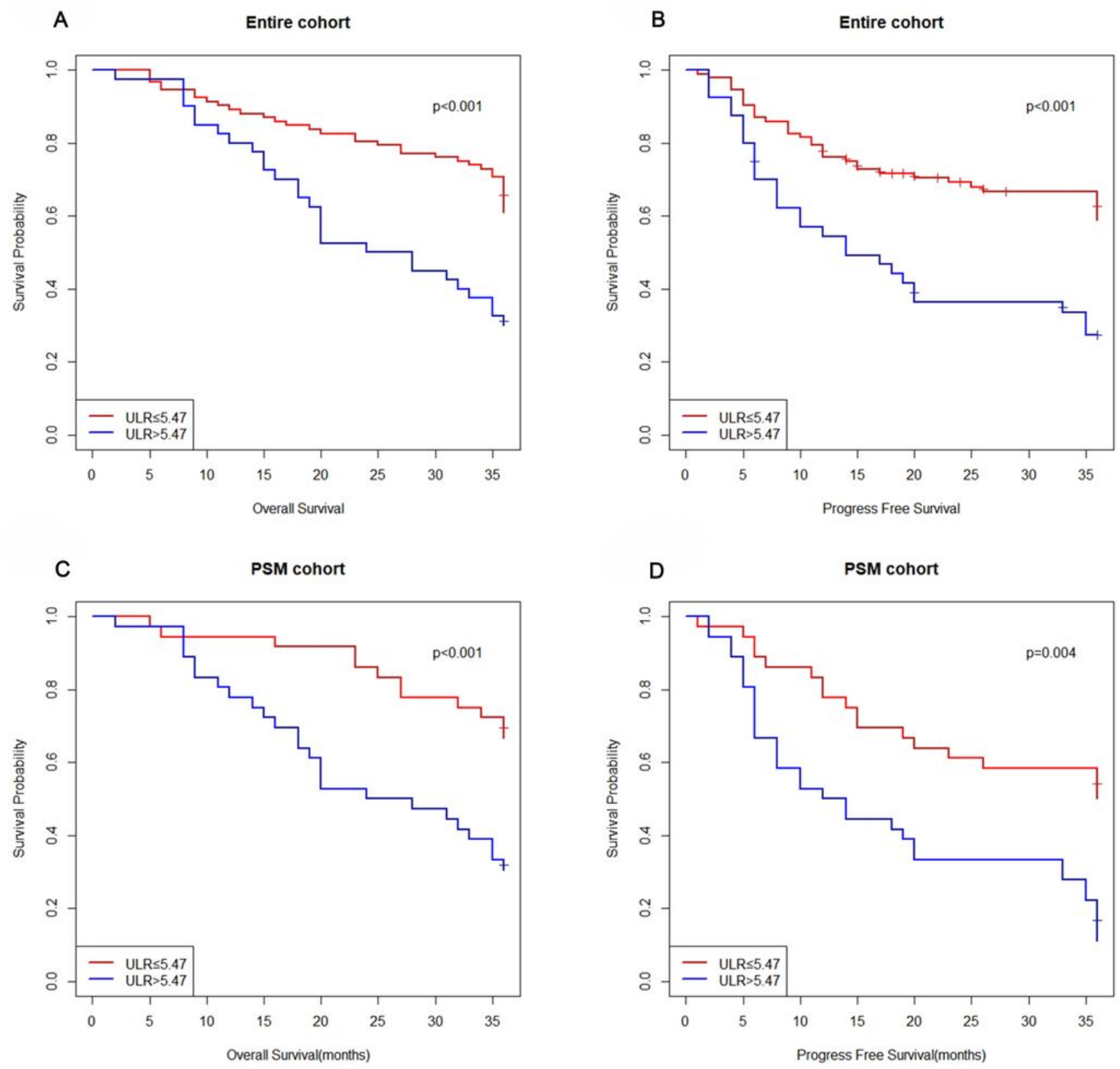

\section{Figure 3}

Kaplan-Meier survival analysis of OS (A) and PFS (B) between the two groups in the entire cohort; KaplanMeier survival analysis of OS (C) and PFS (D) in the PSM cohort. Survival curve showed that both OS and PFS in high-level patient group were significantly shorter than those in low-level group (log-rank $\mathrm{P}<$ 0.001).

\section{Supplementary Files}


This is a list of supplementary files associated with this preprint. Click to download.

- Table1.pdf

- Table2.pdf 